Mobile Communication Systems 
Adviser:

L.F. Turner, BSc, PhD, DSc(Eng), CEng, FIEE, FIERE Professor of Digital Communications Imperial College of Science and Technology London 


\section{Mobile Communication Systems}

J.D. Parsons, DSc(Eng), FEng, FIEE David Jardine Professor of Electrical Engineering University of Liverpool

and

J.G. Gardiner, PhD, CEng, FIEE Professor of Electronic Engineering University of Bradford

\section{Blackie \\ Glasgow and London \\ Halsted Press,}

a division of John Wiley \& Sons, Inc., New York 
Blackie and Son Limited,

Bishopbriggs, Glasgow G64 2NZ

7 Leicester Place, London WC2H 7BP

Distributed in the USA by

John Wiley and Sons, Inc., Now York.

Orders from USA only should be sent to

John Wiley \& Sons, 1 Wiley Drive

Somerset, New Jersey 08873

(C) 1989 Blackie and Son Ltd

Softcover reprint of the hardcover 1 st edition 1989

First published 1989

All rights reserved.

No part of this publication may be reproduced,

stored in a retrieval system, or transmitted,

in any form or by any means,

electronic, mechanical, recording or otherwise,

without prior permission of the Publishers.

\section{British Library Cataloguing in Publication Data}

\section{Parsons, J.D.}

Mobile communication systems

1. Mobile radio systems

I. Title II. Gardiner, J.G.

$621.3841 ' 65$

ISBN-13: 978-0-216-92261-7

Library of Congress Cataloging-in-Publication Data

Parsons, J.D. (John David)

Mobile communication systems/J.D. Parsons, J.G. Gardiner.

p. cm.-(Blackie series in information technology)

Includes bibliographies and index.

ISBN-13: 978-0-216-92261-7 e-ISBN-13: 978-1-4684-1526-1

DOI: 10.1007/978-1-4684-1526-1

1. Mobile communication systems. I. Gardiner, J.G. II. Title.

III. Series.

TK6570.M6P37 1989

$621.3845-\mathrm{dc} 19$ 


\section{Preface}

During the past decade there has been a dramatic change in the nature of mobile communications technology and its impact on the general communications environment. In the 1970s, mobile radio was a minority activity in communications, based on relatively unsophisticated technology. The 1980s, however, have seen the emergence of analogue cellular systems and the definition of future digital systems, and the predicted demand for these services is such that investigations into the use of higher frequency bands have already begun. It is predicted that, by the late 1990 s, the 'personal communications' world will have resulted in the majority of adults in Europe and North America being dependent on radio-connected terminals of various kinds for more than $50 \%$ of their total telecommunications needs. The technology which will form the basis of this revolution has now been defined, at least in outline, and the fixed and mobile equipment that will be used in systems of the future will bear little resemblance to that available even ten years ago.

It is impossible within the confines of a single, relatively short book to cover all the subject areas needed for a study of this exciting and expanding field of technology. We have, perforce, been selective and have chosen those topics which we believe to be of primary importance at the present time. Even within these topics, however, we have been further constrained to concentrate only on aspects directly relevant to mobile communications, an example of this being Chapter 4 which is concerned with modulation. We have concentrated exclusively on land mobile radio as used in the civilian scenario, and have not covered satellite mobile systems or military mobile radio. Although some topics, such as trunking, are important, we have been able to find room for only a brief treatment of the principles; readers requiring further information should consult the references at the end of the relevant chapter.

In the period up to the mid 1970s, both fixed and mobile equipment was fairly primitive and systems engineering was largely concerned with using this equipment to maximize the use of the available radio channels. In modern systems, the radio channel is still a major factor and, following Chapter 1 which gives some background for readers with little prior knowledge, we deal with the properties and characteristics of the channel with regard to both 
signals and noise. We have deliberately chosen to present the information about multipath and short-term effects in Chapter 2, ahead of that on median signal strength prediction and long-term effects in Chapter 3; this seems logical to us, although there may be those who would have preferred to see the order of presentation reversed. Modulation is covered in Chapter 4, before the final chapter on the channel, which deals with noise. Again, opinions may differ on the order of presentation, but we felt that some background on modulation was needed before addressing the problem of how impulsive noise affects communication systems in Chapter 5. Diversity reception, a subject that has been researched for many years, is of increasing importance as mobile systems move into the digital era. Chapter 6 is a summary of the principles and practice which, it is hoped, will pave the way for those who wish to delve deeper into the literature.

A great deal of modern systems engineering now focuses on digital signal processing, channel and speech coding, spectrally efficient modulation methods and, perhaps most importantly, integration of the radio segment into complete systems using fixed-line networks with computer management, and with the public switch telephone network (PSTN). A detailed treatment of these topics is well beyond the scope of this book, but cellular systems embrace them all to a greater or lesser extent. In any case, cellular systems are of fundamental importance in their own right and three chapters at the end of the book are devoted to them: Chapter 7 deals with the fundamentals, Chapter 8 discusses analogue systems, using TACS as the primary example and comparing it briefly with other systems; Chapter 9 introduces the digital cellular systems of the future and shows how the techniques mentioned above influence decisions about channel bandwidth, multiple access techniques and system capacity.

Throughout the book we have, in the interests of brevity and readability, declined to reproduce lengthy mathematical derivations which can, in any case, be found elsewhere. Nevertheless, we have tried wherever possible to use the mathematical notation which is in common usage, to make it easier for readers to understand relevant published papers with a minimum of difficulty.

No book of this kind results from the efforts of only the authors whose names appear on the title page. We have been privileged, over the years, to supervise the research work of many students who have tackled difficult problems, come forward with original ideas, stimulated our thoughts and generally contributed much to our knowledge.- In the context of this book, particular mention must be made of Dr Anwar Bajwa, a former student and academic colleague of J.D. Parsons, and now a valued friend; he contributed much during the early stages of writing, and his influence and advice are gratefully acknowledged. Finally, grateful thanks are due to our wives, Mary and Sheila, who have encouraged us not only in the writing of this book, but throughout our careers; they have made many scarifices which are much appreciated. 


\section{Contents}

1 Introduction to mobile communications 1

1.1 Background 1

1.2 Mobile radio system fundamentals 2

1.2.1 The operating frequency 2

$\begin{array}{ll}1.2 .2 \text { Modulation } & 5\end{array}$

1.3 A simple mobile radio system 5

$\begin{array}{lll}1.4 & \text { Practical communication systems } & 7\end{array}$

$\begin{array}{ll}\text { 1.4.1 Links } & 7\end{array}$

$\begin{array}{ll}1.4 .2 \text { Repeaters } & 8\end{array}$

1.4.3 Talk-through $\quad 8$

1.4.4 Communication with selected mobiles 9

1.4.5 Continuous tone controlled signalling system (CTCSS) 10

$\begin{array}{ll}\text { 1.4.6 Selective calling } & 10\end{array}$

$\begin{array}{lll}1.5 & \text { Paging } & 11\end{array}$

$\begin{array}{ll}\text { 1.5.1 Simple paging systems } & 12\end{array}$

$\begin{array}{ll}1.5 .2 \text { Voice paging system } & 12\end{array}$

$\begin{array}{ll}1.5 .3 \text { Overlay paging system } & 13\end{array}$

$\begin{array}{ll}1.5 .4 \text { Wide-area paging } & 13\end{array}$

$\begin{array}{lll}1.6 & \text { Portables } & 14\end{array}$

$\begin{array}{lll}1.7 & \text { Dialling systems } & 14\end{array}$

$\begin{array}{ll}1.8 & \text { Radiophone services } \\ 1.9 & 15\end{array}$

$\begin{array}{ll}1.9 & \text { Channel sharing } \\ & 15\end{array}$

$\begin{array}{lll}1.9 .1 & \text { Trunking } & 16\end{array}$

1.10 Area coverage techniques 21

$\begin{array}{ll}1.10 .1 \text { Alternative techniques } & 23\end{array}$

$\begin{array}{ll}1.10 .2 \text { Cellular schemes } & 23\end{array}$

$\begin{array}{ll}1.11 \text { Present and future use of mobile radio } & 25\end{array}$

$\begin{array}{ll}\text { References } & 26\end{array}$

2 Multipath characteristics in urban areas 27

$\begin{array}{ll}2.1 \text { Introduction } & 27\end{array}$

2.2 The nature of multipath propagation 29

$\begin{array}{lll}2.3 & \text { Short-term fading } & 32\end{array}$

$\begin{array}{ll}\text { 2.3.1 The scattering model } & 32\end{array}$

$\begin{array}{ll}\text { 2.3.2 Angle of arrival and signal spectra } & 34\end{array}$

$\begin{array}{ll}\text { 2.3.3 Fading envelope statistics } & 35\end{array}$

$\begin{array}{ll}2.3 .4 & \text { Level-crossing rate } \\ 23.5 & 36\end{array}$

$\begin{array}{ll}\text { 2.3.5 Average fade duration } & 37\end{array}$

2.3.6 Spatial correlation of field components 37

2.3.7 Random FM 41

2.4 Frequency-selective fading 43 
2.5 Channel characterization 44

2.6 Channel sounding techniques 48

2.7 Practical channel sounders $\quad 49$

2.7.1 Data processing 51

2.8 Small-area characterization 53

$\begin{array}{ll}\text { 2.8.1 Statistical characterization } & 55\end{array}$

2.9 Large-area characterization $\quad 59$

$\begin{array}{ll}\text { 2.9.1 Distribution of the channel parameters } & 60\end{array}$

$\begin{array}{ll}2.10 \text { Conclusions } & 66\end{array}$

$\begin{array}{ll}\text { References } & 67\end{array}$

3 Propagation and signal strength prediction 68

$\begin{array}{lll}3.1 & \text { Introduction } & 68\end{array}$

3.2 Fundamentals of VHF and UHF propagation $\quad 69$

$\begin{array}{lll}3.2 .1 & \text { Propagation in free space } & 70\end{array}$

$\begin{array}{ll}\text { 3.2.2 Propagation over a reflecting surface } & 71\end{array}$

$\begin{array}{ll}\text { 3.2.3 The effect of surface roughness } & 72\end{array}$

$\begin{array}{lll}3.3 & \text { Propagation over terrain obstacles } & 73\end{array}$

$\begin{array}{ll}\text { 3.3.1 Fresnel zones } & 73\end{array}$

3.3.2 Diffraction over rounded objectacles $\quad 76$

$\begin{array}{lll}3.4 & \text { Multiple knife-edge diffraction } & 79\end{array}$

3.4.1 The Bullington equivalent knife-edge method $\quad 79$

$\begin{array}{ll}\text { 3.4.2 The Epstein-Peterson diffraction loss method } & 79\end{array}$

3.4.3 The Japanese atlas method $\quad 80$

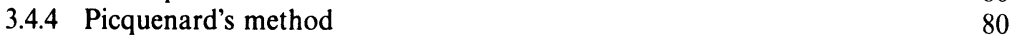

$\begin{array}{ll}\text { 3.4.5 The Deygout method } & 81\end{array}$

$\begin{array}{ll}\text { 3.4.6 Comparison of the diffraction models } & 82\end{array}$

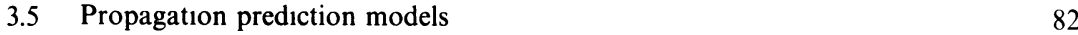

3.6 Signal strength prediction in urban areas $\quad 84$

3.6.1 Allsebrook's method $\quad 86$

3.6.2 Ibrahim's method $\quad 86$

$\begin{array}{lll}3.7 & \text { Discussion } & 89\end{array}$

$\begin{array}{lll}3.8 & \text { Signal variabılity } & 90\end{array}$

3.8.1 Statistical analysis of the signal $\quad 91$

$\begin{array}{lll}3.9 & \text { Large area statistics } & 95\end{array}$

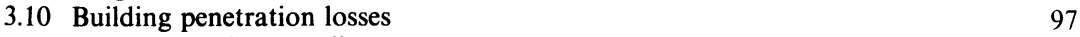

3.10.1 Interference effects 98

References $\quad 99$

4 Modulation techniques $\quad 101$

$\begin{array}{lll}4.1 \text { Introduction } & 101\end{array}$

$\begin{array}{lll}4.2 & \text { Amplitude modulation } & 101\end{array}$

$\begin{array}{ll}\text { 4.2.1 Reduced carrier modulation } & 103\end{array}$

$\begin{array}{ll}\text { 4.2.2 Single-sideband modulation } & 103\end{array}$

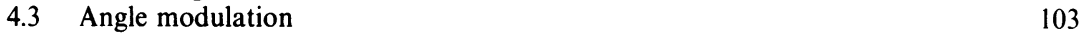

$\begin{array}{ll}\text { 4.3.1 Bandwidth of angle-modulated signals } & 104\end{array}$

4.3.2 Criteria for determining bandwidth 105

$\begin{array}{ll}\text { 4.3.3 Carson's rule } & 105\end{array}$

$\begin{array}{ll}\text { 4.3.4 Narrowband modulation } & 106\end{array}$

$\begin{array}{lll}4.4 & \text { Implementation of AM systems } & 106\end{array}$

$\begin{array}{lll}4.5 & \text { Single-sideband implementation } & 107\end{array}$

$\begin{array}{ll}\text { 4.5.1 Filter method } & 107\end{array}$

$\begin{array}{ll}\text { 4.5.2 Outphasing methods } & 108\end{array}$

4.5.3 Problems in SSB implementation $\quad 110$

$\begin{array}{lll}4.6 & \text { Demodulation } & 112\end{array}$

$\begin{array}{ll}\text { 4.6.1 Coherent demodulation } & 112\end{array}$

$\begin{array}{ll}\text { 4.6.2 Non-coherent (envelope) detection } & 114\end{array}$ 
$\begin{array}{lll}4.7 & \text { Generation of FM signals } & 114\end{array}$

$\begin{array}{lll}\text { 4.7.1 Direct methods } & 115\end{array}$

$\begin{array}{ll}\text { 4.7.2 Indirect methods } & 115\end{array}$

$\begin{array}{lll}4.8 & \text { FM demodulators } & 116\end{array}$

$\begin{array}{lll}\text { 4.8.1 Frequency discriminators } & 116\end{array}$

$\begin{array}{ll}\text { 4.8.2 Pulse-countıng discrımınators } & 118\end{array}$

$\begin{array}{lll}\text { 4.8.3 Phase-locked loops } & 119\end{array}$

$\begin{array}{ll}\text { 4.8.4 Quadrature detection } & 120\end{array}$

$\begin{array}{lll}4.9 & \text { The effect of noise on AM systems } & 121\end{array}$

$\begin{array}{lll}\text { 4.9.1 Noise performance of DSBSC and SSB } & 122\end{array}$

$\begin{array}{ll}\text { 4.9.2 Noise performance of AM } & 122\end{array}$

$\begin{array}{ll}\text { 4.9.3 Comparison of the modulation methods } & 123\end{array}$

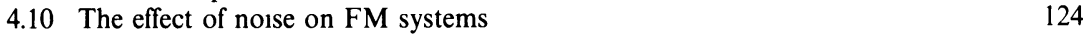

4.10.1 The SNR-bandwidth interchange and FM threshold 124

$\begin{array}{lll}4.10 .2 & \text { Pre-emphasis and de-emphasis } & 126\end{array}$

$\begin{array}{lll}4.11 & \text { The effects of multipath propagation } & 126\end{array}$

$\begin{array}{lll}\text { 4.11.1 Practical SSB systems } & 131\end{array}$

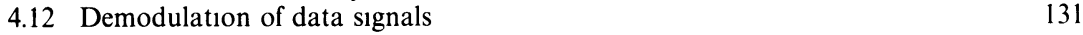

\begin{tabular}{ll}
4.13 & Differentially encoded PSK (DPSK) \\
\hline
\end{tabular}

$\begin{array}{ll}\text { 4.14 The effect of noise in data communication systems } & 135\end{array}$

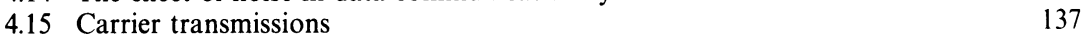

$\begin{array}{ll}\text { 4.16 The influence of multipath fading on data transmissions } & 137\end{array}$

4.16.1 Non-coherent FSK and DPSK 139

$\begin{array}{lll}4.16 .2 \text { Coherent systems } & 140\end{array}$

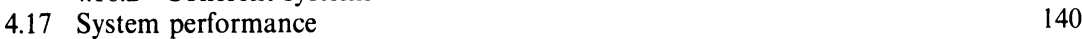

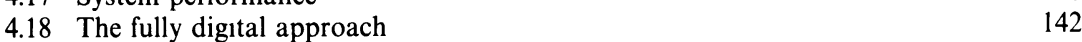

4.18.1 The principal compromises $\quad 142$

$\begin{array}{ll}\text { 4.18.2 Quadrature amplitude modulation } & 143\end{array}$

$\begin{array}{lll}4.18 .3 & \text { Frequency modulation schemes } & 145\end{array}$

$\begin{array}{ll}\text { References } & 147\end{array}$

5 Man-made noise 149

$\begin{array}{lll}5.1 & \text { Introduction } & 149\end{array}$

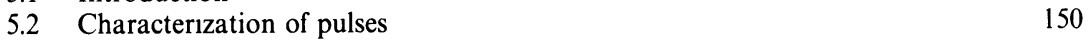

5.3 Characterization of impulsive noise 151

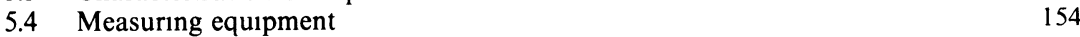

$\begin{array}{ll}\text { 5.4.1 Bandwidth } & 154\end{array}$

$\begin{array}{lll}\text { 5.4.2 Dynamic range } & 155\end{array}$

5.4.3 Sensitivity and noise figure 155

5.4.4 Impulse response considerations 155

$\begin{array}{ll}\text { 5.5 Practical measuring systems } & 157\end{array}$

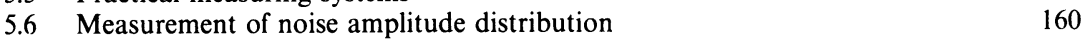

$\begin{array}{lll}5.7 & \text { Statistical characterization of noise } & 161\end{array}$

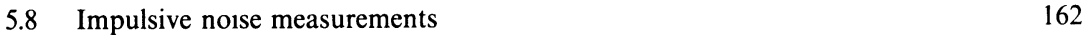

$\begin{array}{lll}5.9 & \text { Summary } & 169\end{array}$

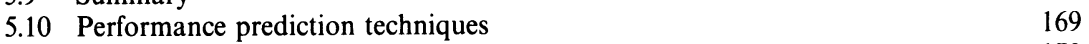

$\begin{array}{lll}\text { 5.10.1 The assessment of receiver performance } & 170\end{array}$

$\begin{array}{ll}\text { 5.10.2 Prediction methods using APD } & 177\end{array}$

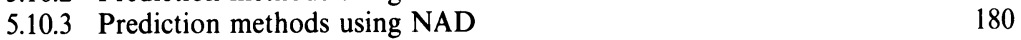

$\begin{array}{ll}\text { 5.10.4 The Bello and Esposito technique } & 182\end{array}$

$\begin{array}{ll}\text { 5.10.5 Application to other receivers } & 184\end{array}$

$\begin{array}{lr}\text { References } & 186\end{array}$

6 Diversity reception 189

$\begin{array}{lll}6.1 & \text { Introduction } & 189\end{array}$

6.2 Basic diversity methods $\quad 190$

$\begin{array}{lll}\text { 6.2.1 Selection diversity } & 192\end{array}$ 
6.2.2 Maximal-ratio combining 194

$\begin{array}{ll}\text { 6.2.3 Equal-grain combining } & 195\end{array}$

$\begin{array}{lll}6.3 & \text { Improvements obtainable from diversity } & 196\end{array}$

$\begin{array}{lll}\text { 6.3.1 Envelope probability distributions } & 197\end{array}$

6.3.2 Level-crossing rate and average fade duration 201

6.3.3 Random FM 203

$\begin{array}{lll}6.4 & \text { Switched diversity } & 205\end{array}$

6.4.1 Cumulative probability distribution 208

$\begin{array}{ll}\text { 6.5 The effect of diversity on data systems } & 211\end{array}$

$\begin{array}{ll}\text { 6.6 Practical diversity systems } & 215\end{array}$

$\begin{array}{lll}6.7 & \text { Predetection diversity } & 216\end{array}$

6.7.1 Phase-sweeping or 'mode averaging' diversity 220

$\begin{array}{lll}6.8 & \text { Diversity systems using special receivers } & 222\end{array}$

6.8.1 Pilot-tone systems 224

6.8.2 Systems without pilot-tone $\quad 225$

$\begin{array}{lll}6.9 & \text { Switched diversity } & 226\end{array}$

6.10 Comparison $\quad 230$

$\begin{array}{lll}6.11 & \text { Postdetection diversity } & 232\end{array}$

6.11.1 Use of a modified phase-correction loop 234

$\begin{array}{ll}6.11 .2 & \text { Unified analysis of postdetection diversity } 235\end{array}$

6.11.3 Postdetection selection and switched diversity 235

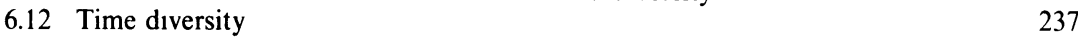

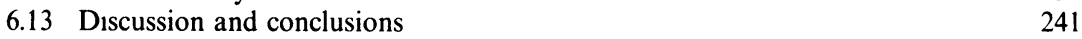

References $\quad 242$

7 Using the radio channel in cellular radio networkgs 244

$\begin{array}{lll}7.1 \text { The radio channel as a system component } & 244\end{array}$

$\begin{array}{ll}7.2 \text { Wideband versus narrowband } & 245\end{array}$

$\begin{array}{ll}7.3 \text { Cellular radio fundamentals } & 245\end{array}$

$\begin{array}{ll}\text { 7.3.1 Why 'cellular'? } & 245\end{array}$

$\begin{array}{lll}\text { 7.3.2 Frequency re-use strategies } & 247\end{array}$

8 Analogue cellular radio systems 253

81 Channel structures 253

$\begin{array}{ll}8.2 & \text { Specifications for the radio equipment } \\ & 255\end{array}$

8.2.1 RF power levels 256

$\begin{array}{ll}\text { 8.2.2 Modulation } & 256\end{array}$

$\begin{array}{ll}\text { 8.2.3 Spectrum and channel designation } & 258\end{array}$

$\begin{array}{lll}8.3 & \text { Network control activity } & 260\end{array}$

$\begin{array}{lll}\text { 8.3.1 Dedicated control channels } & 260\end{array}$

$\begin{array}{ll}\text { 8.3.2 Protecting data messages } & 260\end{array}$

$\begin{array}{ll}\text { 8.3.3 Signalling formats } & 261\end{array}$

$\begin{array}{ll}\text { 8.3.4 Supervision } & 265\end{array}$

$\begin{array}{lll}8.4 & \text { System operation } & 266\end{array}$

8.4.1 Principal functions 266

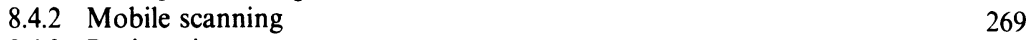

$\begin{array}{ll}8.4 .3 \text { Registration } & 269\end{array}$

$\begin{array}{ll}8.4 .4 \text { Call origination } & 270\end{array}$

$\begin{array}{ll}8.4 .5 \text { Call receipt } & 270\end{array}$

$\begin{array}{ll}8.4 .6 \text { Hand-off } & 270\end{array}$

$\begin{array}{ll}\text { 8.4.7 Call termination } & 271\end{array}$

$\begin{array}{lll}8.5 \text { Some system comparisons } & 271\end{array}$

9 Digital cellular radio systems 273

9.1 Digital versus analogue for second-generation cellular systems 273

$\begin{array}{ll}\text { 9.2 Choice of basic system architecture } 275 & 273\end{array}$ 
9.2.1 Single channel per carrier, FDMA 275

$\begin{array}{ll}\text { 9.2.2 Time division multiple access } & 276\end{array}$

$\begin{array}{ll}9.3 \text { Essential techniques for digital implementation } & 277\end{array}$

$\begin{array}{ll}\text { 9.3.1 Speech coding } & 277\end{array}$

$\begin{array}{ll}\text { 9.3.2 Channel coding } & 280\end{array}$

$\begin{array}{ll}\text { 9.3.3 Channel equalization } & 281\end{array}$

$\begin{array}{ll}\text { 9.3.4 Frequency hopping } & 283\end{array}$

$\begin{array}{lll}9.4 & \text { Example systems } & 284\end{array}$

$\begin{array}{ll}\text { 9.4.1 An asymmetrical system-MATS-D } & 284\end{array}$

\begin{tabular}{ll} 
9.4.2 A wideband TDMA system-CD900 & 285 \\
\hline
\end{tabular}

9.4.3 The GSM system-narrowband TDMA 286

$\begin{array}{lll}9.5 & \text { Postscript } & 288\end{array}$

Index $\quad 289$ 